\title{
Ricerca storica e cartografica delle domus federiciane "fantasma" della regione del Principatus et Terra Beneventana
}

\author{
Historical and cartographic research about the ghost domus built by Frederick II in Principa- \\ tus et Terra Beneventana region
}

\author{
Antonella Furno \\ Università degli Studi Suor Orsola Benincasa, Naples, Italy, antonella.furno@ hotmail.it
}

\begin{abstract}
During his reign Frederick II built a series of representative fortified constructions in southern Italy, and after reinforcing the defence line of the border with the State of the Church, he decided to build many residential estates called domus or palacium in the fundamental medieval textual source of Statutum de reparatione castrorum. This research is focused on the study of the landscape in the ancient region of Principatus et Terra Beneventana during the thirteenth century: it is noticed the presence of five imperial domus cited in the Statutum with the name domus Castellucci Battipallae, castrum et palacium Sarni, domus imperatoris in Ebulo, domus imperatoris Apicii and the Castel Belvedere Marano palace. Every domus was studied through a historical and cartographic analysis, and in case of the structure is not recognised on the territory it was organized a landscape analysis in order to propose a hypothetical position. The data that was gathered into ArcGIS software to define the probable locations of the ghost domus were the detailed routes of ancient roads related to the positions of the casalia (little rural communities that paid taxes to maintenance of the royal structures), the Church properties, the urban site, and the other castra and domus.
\end{abstract}

Keywords: Southern Italy, Frederick II, late medieval landscape, GIS.

\section{Introduzione}

Federico II rientrò in Italia nel 1220 e polarizzò l'attenzione nelle province continentali dell'Italia meridionale, con un chiaro progetto di edificazione di una serie di strutture architettoniche con chiaro scopo difensivo. Successivamente si dedicò alle cosiddette domus solatiorum nelle retrovie del regno, strutture con valore non squisitamente difensivo, ma luoghi legati allo svago e al piacere (Bertaux, 1903; Haseloff, 1920; Sthamer, 1995). Consultando la fonte angioina de lo Statutum de reparatione castrorum, è facile rintracciare le denominazioni degli edifici a destinazione residenziale presenti nella regione del Principatus et Terra Beneventana, in quanto riportati esplicitamente nel catalogo con i termini domus o palacium (e quindi non castrum). Le strutture citate sono: la domus Castellucci Battipallae, castrum et palacium Sarni, domus imperatoris in Ebulo, domus imperatoris Apicii e la domus di Castel Belvedere di Marano, menzionata solo in versioni più tarde (Sthamer, 1995, pp. 110, 111, 159). 


\section{Analisi delle fonti e del paesaggio}

Dall'analisi della preziosa fonte dello Statutum de reparatione castrorum emergono dati interessanti sul rapporto tra castelli e territorio. $\mathrm{La}$ studiosa Gina Fasoli, la quale per prima ha realizzato una collocazione, rimasta purtroppo incompleta, sulla carta topografica dei singoli castelli e delle comunità tenute alla loro manutenzione, è arrivata a delle interessanti constatazioni. È infatti importante notare che le comunità tenute alla manutenzione dei castelli, riportate sullo Statutum, non erano quelle situate nei dintorni immediati e quindi più vicine. Ne sono alcuni esempi il castello di Bari che doveva essere riparato dalle comunità di Rutigliano, Noia e Polignano, rispettivamente distanti dal centro, in linea d'aria, 16,14 e 30 $\mathrm{km}$. Altro esempio il castello di Canosa che doveva essere riparato dagli abitanti di Minervino, a poco più di $15 \mathrm{~km}$ dal centro e da quelli di Giovinazzo, a più di $50 \mathrm{~km}$ in linea d'aria (Houben, 2005). La Fasoli osservò che le comunità tenute alla manutenzione dei castelli, nella maggior parte dei casi, sono "collocate in un fascio che si allarga a ventaglio in una sola direzione, che si estende a molti chilometri in linea d'aria (venti e oltre), distanza che sul terreno doveva aumentare notevolmente, superando dislivelli e attraversando fiumi". La spiegazione di questo fenomeno va senz'altro individuata "attraverso una serie di ricerche sistematiche sull'intersecarsi e sovrapporsi di terre e di diritti demaniali e feudali" (Fasoli, 1980). E' da aggiungere a questa ipotesi che la collocazione delle comunità tenute alla manutenzione è fortemente condizionata da fattori geografici. Inoltre si ritiene che questa ubicazione possa essere dovuta anche a una logica politica, cioè alla volontà, da parte di Federico II, di evitare che una struttura castellare, con la sua guarnigione e il suo castellano, potesse raccordarsi troppo strettamente alle comunità di quel territorio, sino a diventare pericoloso centro di aggregazione di interessi comuni. Sulla base di queste intuizioni, si è proceduto a creare un modello predittivo in ambiente GIS sui casi analizzati nella regione del Principato, partendo dalla denominazione delle strutture dello Statutum. Di queste, le domus di Marano e di Apice sono state collegate con certezza a strutture architettoniche presenti sul territorio, quella di Sarno e Battipaglia legate a realtà castrensi ma non individuabili in strutture ben definite, mentre la posizione della domus di Eboli resta completamente sconosciuta.

Di seguito si riportano i casi analizzati.

\section{-Domus Castellucii Battipalie}

La struttura di Battipaglia viene reparata dagli uomini del casale Castelluccio, di San Mattia, del Ponte di Tussiano, di Santa Cecilia, di Olevano, di Montecorvino e di Acerno (Sthamer, 1995, p. 110).

Per individuare su cartografia contemporanea i casalia che devono reparare le domus, è stato utilizzato un file shape dei comuni d'Italia attuali unito ad un ulteriore file shape dei centroidi dei comuni italiani (centro geometrico rispetto alla geometria del comune), con un riferimento ai microtoponimi secondo l'ipotesi di Sthamer alla cartografia storica di Rizzi Zannoni (Rubbettino, 1994) e, ovviamente alla letteratura pregressa edita riguardo il paesaggio medievale del XIII secolo.

Il casale di Castelluccio viene ricordato nelle fonti, strappato alla Chiesa Salernitana dal conte svevo Marcovaldo passato, poi ai Cavalieri Teutonici e alla morte dell'imperatore restituito all'arcivescovo che ne deteneva il possesso fino ai tempi di Roberto il Guiscardo. Nel documento suddetto del 1251 ci informa della cappella e delle terre coltivate nel casale che aveva a controllo un fortilizio. Nel documento si riportano i nomi dei 23 capofamiglia degli homines predicti casalis che all'atto della restituzione del castello e del casale prestano giuramento di fedeltà all'arcivescovo. Con questo giuramento di fedeltà alla Chiesa Salernitana questi uomini si dichiaravano "semper vassalli iuris et dictionis dicti archiepiscopi et successorum eius et predicte ecclesie, et gaudeant illa libertate, qua alii vassali ipsius ecclesie gaudere noscuntur" ovvero i vassalli di Battipaglia godranno della libertas di cui godono i vassalli della Chiesa Salernitana (Muro, 2005). 
La chiesa di San Mattia nel 1053 è conosciuta come "quam dominus Guaimarius princeps a nobo fundamine construxit in loco Tusciano" (Leone-Vitolo, 1984, VII, 203) fondata dal principe Guaimario nei pressi della riva sinistra del Tusciano all'incrocio della via che dal mare conducevano a Battipaglia con la via antiqua che proveniva da Santa Cecilia. Oggi rimane solo la località omonima di S. Mattia a qualche chilometro da Battipaglia. San Mattia fu donata insieme alle sue pertinenze all'abate di Cava Pietro nel 1089 dal duca Ruggiero figlio di Roberto il Guiscardo. Dopo il XII secolo San Mattia si configura come una comunità monastica a capo della quale vi è un preposito, mentre nel XIII secolo è un priore a gestire il cenobio (Muro, 2005, p. 31).

Il nucleo del villaggio che si riferisce agli homines Pontis Tussiani probabilmente si riferisce ad un casale nei pressi del suddetto ponte del Tusciano lungo l'antico percorso della via Popilia.

L'altro casale nella piana del Sele è quello di Santa Cecilia. Menzionato in un documento del 1095 (Carlone, 1988, p. 56) in cui Ruggiero, figlio di Guglielmo Trincanotte, per la salvezza della sua anima e di quelle del padre Guglielmo, della madre Lizilia e dei nonni Raone e Emma dona al monastero di San Pietro di Eboli (extra et prope castrum Eboli) retto dall'abate Gregorio, padre spirituale del donatore, la chiesa di San Nicola di Mercatello con tutti i beni pertinenti, l'iscla di Santa Cecilia con tutti i beni mobili e stabili in essa contenuti, l'uso del fiume Sele e tutti gli uomini residenti o che vi avessero risieduto. Santa Cecilia come tutti gli altri casali prende il nome dalla chiesa omonima ricordata nel documento del 1160 in territorio ebulensi...ecclesia S. Cecilia (Giordano, 2014, pp. 94-97) confermata dall' Arcivescovo Romualdo in possesso all'Abate di S. Pietro di Eboli. Probabilmente una via antiqua percorreva la piana proveniente dal Tusciano e costituiva l'asse principale delle comunicazioni del casale sebbene bisogna immaginare una situazione di impaludamento dell'area del Sele. Con Olevano ci si riferisce probabilmente all'agglomerato legato al castrum Olibani, complesso fortificato che si trova a 700 m.s.l.m. nel comune attuale di Olevano sul Tusciano. Tre cinte murarie che limitano tre aree differenziate, alcune aree sono state indagate con scavi archeologici (Muro, 2005). Nel 1230 Federico II confisca il castrum Olibani alla Chiesa Salernitana e ne fa un castrum del demanio regio sotto diretto controllo dell'imperatore e lo affida ad Ermanno di Salza; solo nel 1255 verrà riconsegnato alla Chiesa. Non disponiamo di fonti archivistiche sufficienti legate ai casalia Montis Corbini e Acerni quindi vengono individuate su cartografia con i centroidi dei comuni attuali con toponimo affine (Montecorvino Rovella e Acerno) (Fig. 1).

\section{-Castrum Sarni et palacium domini imperatoris}

La struttura del palacium di Sarno che si trova all'interno della struttura del castrum Sarni deve essere reparata da homines s.Georgii, Braciliani, Furini et Montis fortis (Sthamer, 1995, p. 111). I toponimi riportati nello Statutum vengono attribuiti da Sthamer ai toponimi attuali di Castel San Giorgio, Bracigliano, Forino, Monteforte Irpino individuabili in cartografia con il centroide dei comuni attuali. Le zone di buffer sono state calcolate in base alla distanza più vicina rispetto al polo attrattore del palacium Sarni. Come si vede dalla Figura 2, i centroidi dei comuni attuali di Castel San Giorgio, Bracigliano, Forino, Monteforte Irpino si irradiano rispetto alla struttura del $c a$ strum Sarni in uno schema a ventaglio confermando l'ipotesi della Fasoli. Dopo una lunga ricerca di ricognizione di superficie sul campo, sono stati individuati su cartografia i punti noti dei casalia Santa Cecilia, Castrum Olibani e San Mattia, con i relativi buffer della zona d'influenza. Si sono poi individuati per coadiuvare la ricerca i centroidi dei comuni attuali di Montecorvino Rovella e Acerno. Le zone di buffer sono state calcolate in base alla distanza più vicina rispetto al polo attrattore della $d o$ mus. Il modello a ventaglio viene parzialmente rispettato, soprattutto laddove i punti noti hanno una localizzazione certa (Fig. 2). 

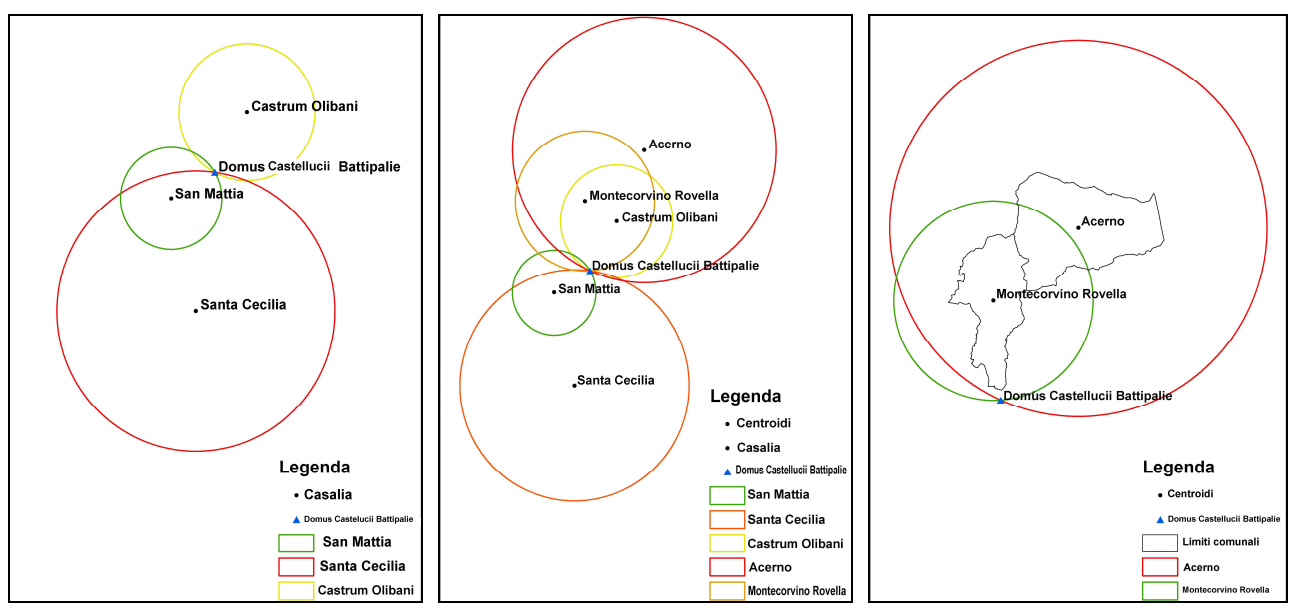

Fig. 1. a-b-c.

\section{La domus "fantasma": il caso della domus imperatoris in Ebulo}

Rappresenta invece un'eccezione la domus di Eboli, in quanto non riconoscibile in nessuna struttura architettonica su territorio, ed essendo legata solo ad unico casale (Olivetum), la posizione non risulta ipotizzabile nemmeno restringendo l'area intorno ai casalia addetti alla reparatione. E' possibile però restringere l'area tenendo in considerazione i territori feudali e del demanio regio circostanti.

L'area che comprende ora i comuni odierni di Olevano sul Tusciano e Battipaglia (dai monti fino alla costa) viene ricordata nelle fonti del $\mathrm{X}$ secolo come locus Tuscianus in finibus salernitanis, quindi di pertinenza amministrativa del principato longobardo di Salerno. Con l'istituzione della signoria del Castrum Olibani si determinò un'ulteriore divisione dell'area, e la parte meridionale verso Salerno venne denominata locus Tuscianus, mentre la parte settentrionale prese la denominazione dal castrum di appartenenza. La situazione non mutò fino al 1230 quando con i contrasti tra Federico II e Chiesa videro la requisizione del castrum Olibani da parte dell'imperatore. Eboli invece, dalle fonti anteriori all'XI secolo viene menzionata come locus all'interno delle dipendenze amministrative di Salerno. Nel 1047 è ricordata come castellum, centro di un territorio ben definito gestita dalla famiglia del conte
Lamberto. All'indomani della conquista del Principato da parte di Roberto il Guiscardo si ebbe il riconoscimento del nuovo dominus di Eboli incardinato nella vasta Contea di Principato a capo della quale era Guglielmo d'Altavilla (Carlone, 1988, pp. 217-220). Successivamente con la guerra tra Enrico VI e i Normanni nella quale Eboli si schierò con gli svevi, conclusasi con la vittoria dell'imperatore portò a una ridefinizione del controllo della contea che fu restituita nel 1195 dal figlio di Adelasia (figlia naturale di Ruggero II). Nel 1219 Federico II immise la terra e gli abitanti di Eboli nel regio demanio e quindi sotto il controllo diretto della Corona. Dalle fonti si legge la presenza prima di un catapano $(1216,1220)$ poi dal 1224 di un baiulo. Alla morte di Federico, nel 1250 Manfredi, immemore dell'ammonimento paterno che lo consigliava di non smembrare il demanio del regno, diede in feudo a Giordano Lancia la terra di Eboli. Giordano tenne il feudo per un breve periodo, ma ebbe la possibilità di estendere la reale riserva di caccia tenuta dal re Manfredi aggiungendo un'altra selva che prima era aperta agli usi civici dei cittadini di Eboli.

Alla morte di costui, con gli Angioini la terra di Eboli ritornò nuovamente al demanio regio (Longobardi, 1998, p. 142). La delimitazione territoriale del comitato di Eboli non è di facile definizione, probabilmente la circoscrizione in epoca normanna ricalcava quella del Principato 

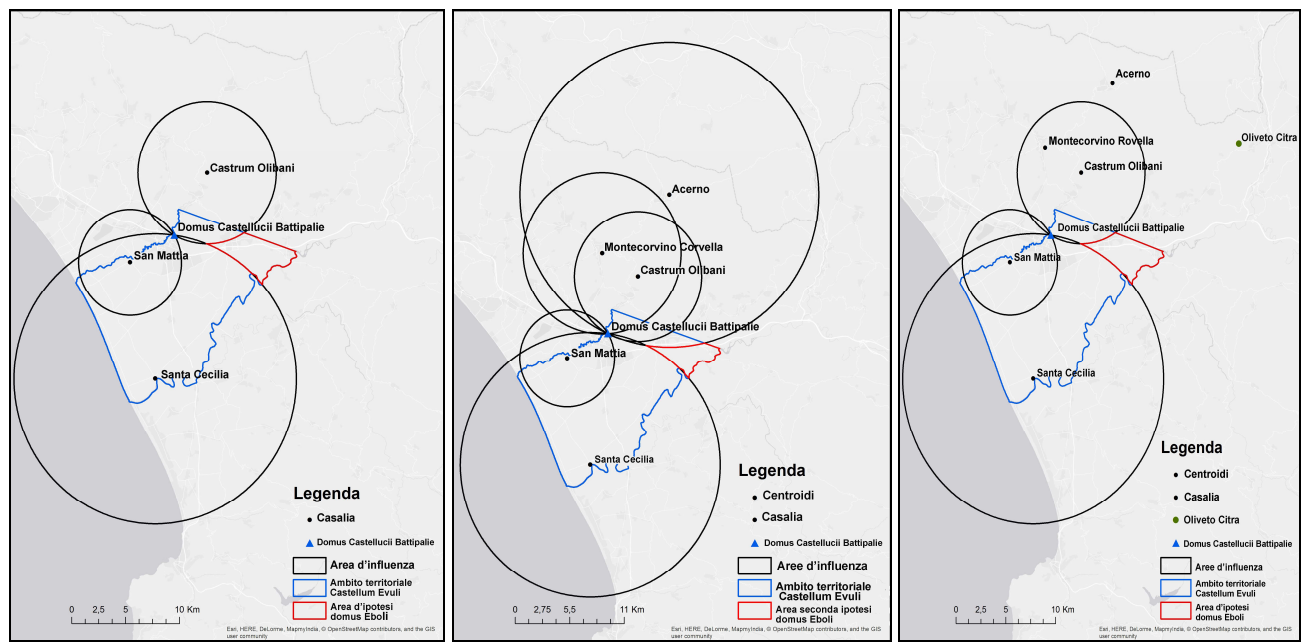

Fig. 2. a-b-c.

longobardo di Salerno; probabilmente come ipotizza Di Muro negli ultimi decenni dell'età longobarda si riconsiderarono i confini di Salerno perché considerati troppo ampi da gestire e vennero creati i comitati di Giffoni ed Eboli (Muro, 2001). Nelle fonti viene citato un $\mathrm{ca}$ stellum Evuli, probabilmente da far coincidere con la realtà castrense visibile ancora oggi sul versante meridionale di una bassa collina a controllo della Piana del Sele, della via Popilia e di percorsi che risalivano verso le valli del Tusciano, del Sele e l'agro giffonese da una parte e verso Capaccio e quindi il Cilento dall'altra. Dalle fonti di XI secolo si configura come borgo fortificato definito da una cinta muraria e con un complesso abitativo all'interno, un mercato e diversi sobborghi al di fuori delle mura (Muro, 2005, pp. 62-65). La grande crescita dell'insediamento suburbano di Eboli individuato nel XII secolo costituisce insieme a un incremento demografico, una prova di attrazione esercitato dal borgo tale da far risultare gli spazi intramurari non più idonei. Probabilmente un aumento della popolazione dato dai coltivatori che nelle campagne praticavano l'artigianato e scorgevano una possibilità di trovare nel commercio una modalità di ascesa economica.

Probabilmente il contesto dove sorse la domus imperiale di Federico II fu diverso da questo contesto suburbano, o se la struttura federicia- na fosse stata compresa all'interno di questa realtà castrense doveva essere collegata ad una realtà boschiva, la defensa nominata nelle fonti. Dallo Statutum de reparatione castrorum viene menzionata la struttura come Domus domini imperatoris in Ebulo che deve essere reparata da gli uomini della stessa terra e quelli di Oliveto (homines eiusdem terre et per homines Oliveti). Nel 1240 Federico II ordina di rinchiudere alcuni ebolitani che contra mandatum nostrum erano stati trovati a cacciare nelle difese della corte di Eboli. La foresta riservata all'imperatore era vista come un ottimo bottino per far legna, ma sorpresi dai guardiani vennero puniti. Nel 1277 nell'elenco delle defensae regie fatto redigere da Carlo I troviamo anche quella di Eboli e continua ad essere nell'elenco del demanio regio anche successivamente (I Registri della Cancelleria angioina ricostruiti da Riccardo Filangieri con la collaborazione degli archivisti napoletani, Napoli, voll. I-L, [1951-2010], XIX, pp. 155157).

Con questi presupposti, si è ricreato in ambiente GIS un modello per l'analisi spaziale delle aree di influenza della domus di Battipaglia, del castellum Eboli, del castrum Olibani e della domus di Eboli per tentare un'ipotesi di localizzazione di quest'ultima. Le pertinenze amministrative del castellum Ebuli sono state disegnate con la tool Editor di ArGIS con un 
limite occidentale al corso del fiume Cornea fino alla confluenza del Tusciano e quindi a ovest fino alla costa (Muro, 2005, p. 16), grossomodo comprendono i territori dei comuni odierni di Olevano sul Tusciano, Battipaglia ed Eboli. Il limite orientale è stato posto alla foce del Sele sul Tirreno dall'innesto del Fiume La Tenza nei pressi del Ponte Popilia. Il limite settentrionale è "alle pendici sud orientali dei Monti Picentini in corrispondenza dei Monti Maiano e Monte Raione" (Muro, 2005, p. 16).

\section{Conclusioni}

Dalle immagini si nota che i casalia con punti noti non solo mantengono lo schema a ventaglio della Fasoli, ma rientrano nella zona d'influenza della Castelluccia al confine con la buffer zone del castellum Ebuli. Aggiungendo allo schema grafico i punti legati ai centroidi dei comuni attuali di Montecorvino Rovella e Acerno (quindi dalla posizione storica non certa), lo schema a ventaglio viene leggermente mutato e la zona d'influenza si allarga spropositatamente. La zona evidenziata che va oltre i confini sia della Castelluccia sia del Castellum Evuli potrebbe essere intesa come la fascia dell'inizio della zona d'influenza della domus Eboli, non identificata in una determinata struttura architettonica. A conferma di ciò è possibile notare la direzione rispetto al casale che la reparava di Oliveto Citra completamente spostata a nord-est rispetto alle zone citate. L'ipotesi quindi della zona messa in luce risulterebbe attendibile, e con una futura prossima ricognizione sul campo potrebbe esser confermata.

\section{Bibliography}

Bertaux, E. (1903). L'art dans l'Italie méridionale de la fin de l'Empire romain à la Conquête de Charles d'Anjou, II, Parigi (riprod. anast. Parigi-Roma 1968).

Carlone, C. (1988). Documenti per la storia di Eboli, I-II-III, Salerno.

Fasoli, G. (1980). "Castelli e strade nel Regnum Siciliae. L'itinerario di Federico II", in Romanini, A.M., ed., Federico II e l'Arte del duecento italiano (Atti della III settimana di studi di storia dell'arte medievale dell'Università di Roma, 15-20 maggio 1978), Galatina, pp. 27-52.

Giordano, A. (2014). Le pergamene dell'archivio diocesano di Salerno (841-1193), Battipaglia.

Haseloff, A. (1920). Die Bauten der Hohenstaufen in Unteritalien, Leipzig; Calò Mariani, M.S., trad., (1992), Architettura sveva in Italia Meridionale, Bari.

Houben, H. 2005. Domus in Enciclopedia fridericiana, pp. 479-480

Muro, A. Di. (2001). Le terre tra Salerno e il Sele in età longobarda, Salerno.

Muro, A. Di. (2005). La Piana del Sele in età normanno-sveva. Società, territorio e insediamenti. (ca. 1070-1262), Salerno.

Leone, S.; Vitolo, G. (1984). Codex diplomaticus Cavensis, Badia di Cava, vol. IX (1065-1072).

Longobardi, C. (1998). Eboli tra cronaca e storia, Salerno.

Rubbettino, M.P., ed. (1994). Atlante geografico del Regno di Napoli di Giovanni Antonio Rizzi Zannoni, Italia meridionale quadro d'unione, Napoli.

Sthamer, E. (1995). L'amministrazione dei castelli nel Regno di Sicilia sotto Federico II e Carlo I d'Angiò, Bari. 